\title{
Acute Type B Aortic Pathology Mimicking Acute Type A Intramural Hematoma with Organ Malperfusion
}

\author{
Eli Levy, MD¹, Amit Korach, MD¹, Chen Rubinstein, MD², Dan Gilon, MD³, Oz M. Shapira, MD ${ }^{1 *}$ \\ ${ }^{1}$ Department of Cardiothoracic Surgery, “Hadassah” Hebrew University Medical Center, Jerusalem, Israel \\ ${ }^{2}$ Department of Vascular Surgery, "Hadassah" Hebrew University Medical Center, Jerusalem, Israel \\ ${ }^{3}$ Department of Cardiology, "Hadassah" Hebrew University Medical Center, Jerusalem, Israel
}

\begin{abstract}
The management of acute Stanford Type A intramural hematoma (IMH) of the aorta remains controversial. Most surgeons advocate emergency surgery in a manner similar to frank acute Type A dissection. Others recommend a conservative approach to this distinct clinicopathological entity. We describe a case of acute aortic pathology initially diagnosed as Type A IMH with organ malperfusion, subsequently identified as acute Type B pathology with retrograde and antegrade extension. An endovascular approach was successfully used to exclude the site of origin.

Copyright $\odot 2015$ Science International Corp.
\end{abstract}

\section{Key Words}

Aortic dissection • Aortic surgery

\section{Introduction}

Aortic intramural hematoma (IMH) is pathologically distinct from aortic dissection, characterized by hemorrhage into the aortic media in the absence of an intimal tear and direct flow communication between the true and false lumens. Krukenberg [1] suggested that rupture of the vasa vasorum initiates the process of IMH. Gore suggested that medial degeneration predisposes the vasa vasorum to rupture and hemorrhage [2].
The management of acute Stanford Type A intramural hematoma (IMH) of the aorta remains controversial. Most surgeons advocate emergency surgery in a manner similar to frank acute Type A dissection. Others recommend a conservative approach to this distinct clinicopathological entity. We describe a patient with acute Type $B$ aortic pathology mimicking Type A IMH with organ malperfusion that was successfully managed by thoracic endovascular aortic repair (TEVAR).

\section{Case Presentation}

A 56-year-old white female with a history of hypertension, heavy smoking, and chronic obstructive pulmonary disease was admitted to another hospital complaining of severe chest and abdominal pain. Electrocardiogram showed nonspecific ST-T changes. Initial troponin I levels were within normal limits. The patient was transferred immediately for cardiac catheterization with a presumed diagnosis of acute coronary syndrome. Coronary catheterization demonstrated external compression and distortion of the left main coronary artery with no discrete coronary stenoses. The patient was transferred to the acute cardiac care unit where 2-D echocardiogram showed moderate-to-severe left ventricular

* Corresponding Author:

Oz M. Shapira, MD

Department of Cardiothoracic Surgery

Hadassah, Hebrew University Medical Center

POB 12000, Ein Kerem, Jerusalem, 91120, Israel

Tel: +972 2677 6960, Fax: +972 2643 8005, E-Mail: ozshapira@hadassah.org.il 
dysfunction, normal right ventricular function, no significant valvular abnormalities, and suspected acute aortic dissection.

Shortly thereafter, the patient deteriorated into cardiogenic shock with florid pulmonary edema. The patient was sedated and intubated, and inotropic support was initiated and an intra-aortic balloon pump was inserted.

After stabilization, a CT angiogram of the chest and abdomen was obtained. This test was interpreted as Type A IMH starting at the sinotubular junction and extending down to the iliac bifurcation (Figure 1). The IMH was complicated by external compression of the left main coronary artery, as well as liver, right kidney, and colon malperfusion. A large intercostal artery was observed in the proximal descending thoracic aorta (Figure 2). Correspondingly, metabolic acidosis and marked elevation of liver enzymes and international normalized ratio (INR), were observed. Cardiac surgery consult was obtained, but the patient was deemed to be too ill to undergo a major aortic procedure. The initial course was further complicated by Gram-negative sepsis, likely originating from a right lower lobe pulmonary infiltrate. At this time point, this desperately ill patient was transferred to our care.

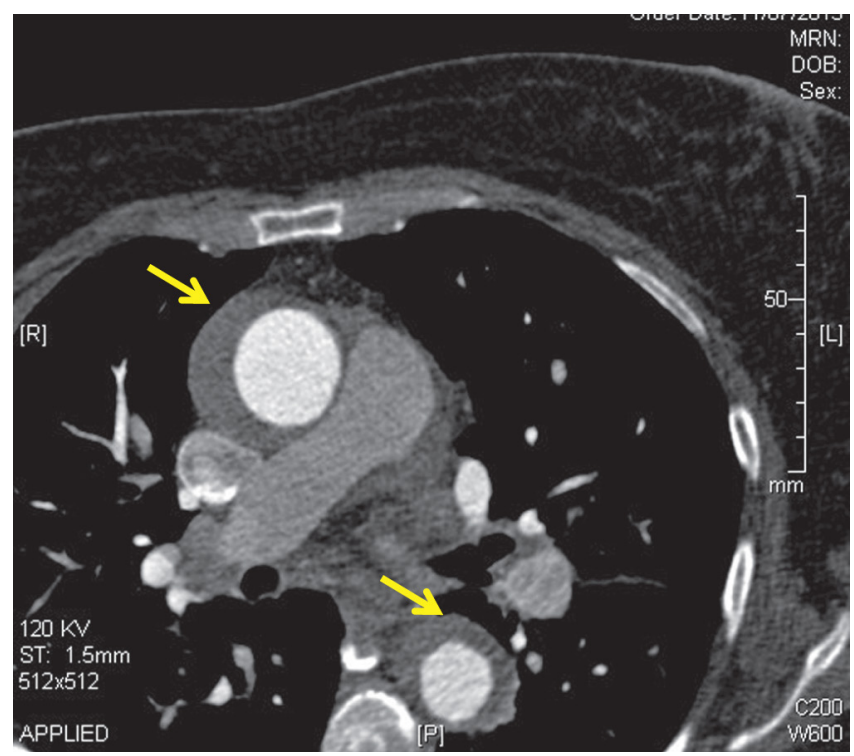

Figure 1. CT angiography axial view demonstrating the aortic intramural hematoma in the ascending and descending aorta (yellow arrows).
After initial stabilization, to better strategize treatment plan, we elected to repeat the imaging studies, 5 days after initial presentation. A transesophageal echocardiogram showed moderate left ventricular dysfunction, moderate aortic insufficiency, extensive Type A IMH, and a large left pleural effusion. Repeat CT angiography demonstrated stable hematoma in the ascending aorta (Figure 3), a newly developed pseudoaneurysm originating within an intimal ulcer in close proximity to the noted large intercostal artery in the proximal descending aorta (Figures 4 and 5) and a new large pleural effusion.

The diagnosis was revised to acute Type B aortic pathology with proximal and distal extension, and we elected to proceed with TEVAR aiming to exclude the intimal tear. Using routine endovascular techniques, a 26×100 mm C-Gore Tag-R Type (W L Gore \&

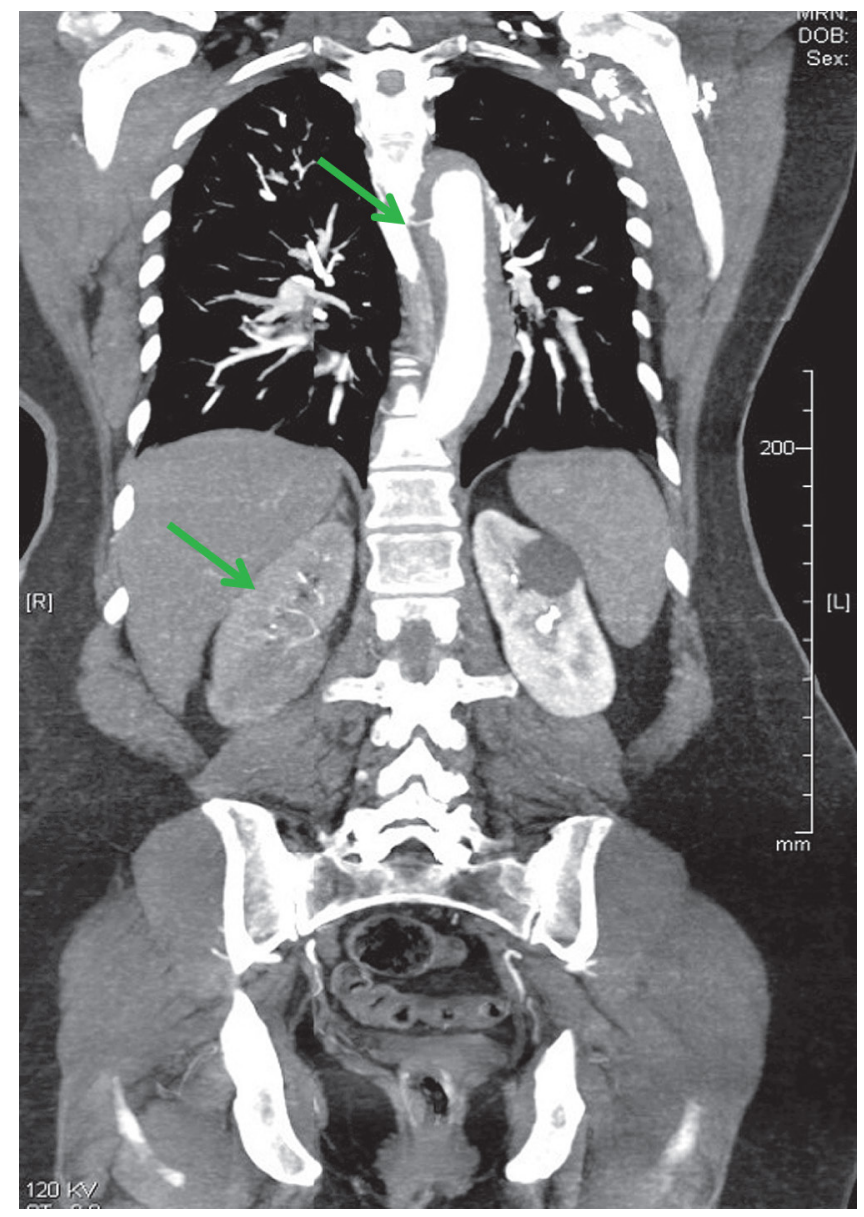

Figure 2. CT angiography coronal view demonstrating the intramural hematoma, large intercostal artery (upper green arrow) and unperfused right kidney (lower green arrow). 


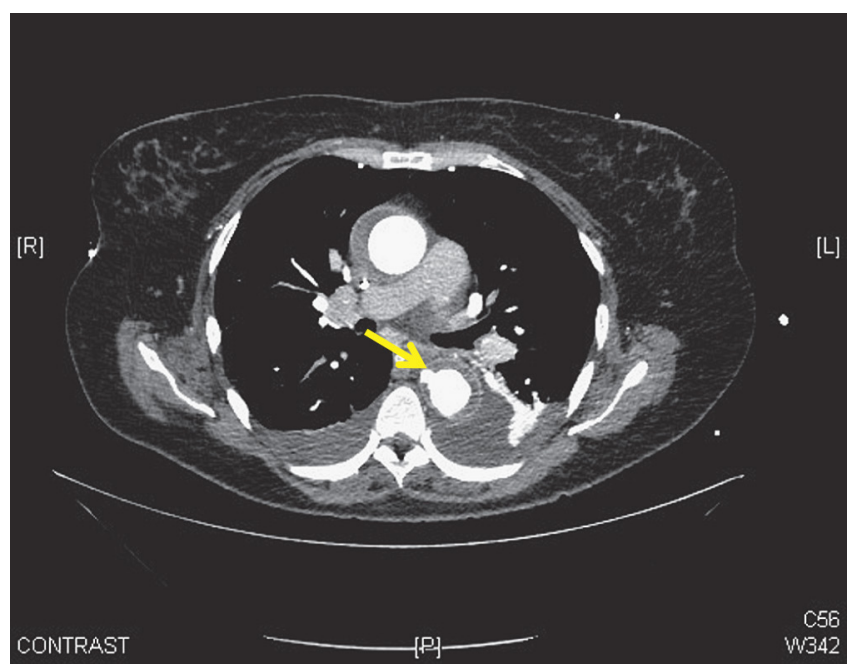

Figure 3. $C T$ angiography axial view demonstrating the pseudoaneurysm (yellow arrow) with extravasation of dye into the hematoma.

Assoc. Inc. Flagstaff, AZ, USA) stent was successfully implanted via the left femoral artery. Due to the fact that the procedure was performed in an acute dissection phase, the balloon at both ends of the stent was not inflated, and an oversized stent was not used.

The patient was extubated 3 days after the procedure. The patient fully recovered and was discharged home on the thirteenth postoperative day. Predischarge CT angiography showed a well-positioned stent graft, no endoleak, and marked decrease in the ascending aortic IMH as well the descending aortic pseudoaneurysm. Thirteen months after surgery, the patient is asymptomatic. Follow-up CT angiography showed complete resolution of the IMH, elimination of the intimal ulcer and remodeling of the pseudoaneurysm (Figures 6, 7, and 8).

\section{Discussion}

Emergency surgery is nearly always recommended in patients with acute Type A aortic pathology and organ malperfusion. The most important principle of surgical repair of typical Type A dissection includes resection and reconstruction of the aortic segment containing the intimal tear initiating the dissection. This is most commonly achieved via a

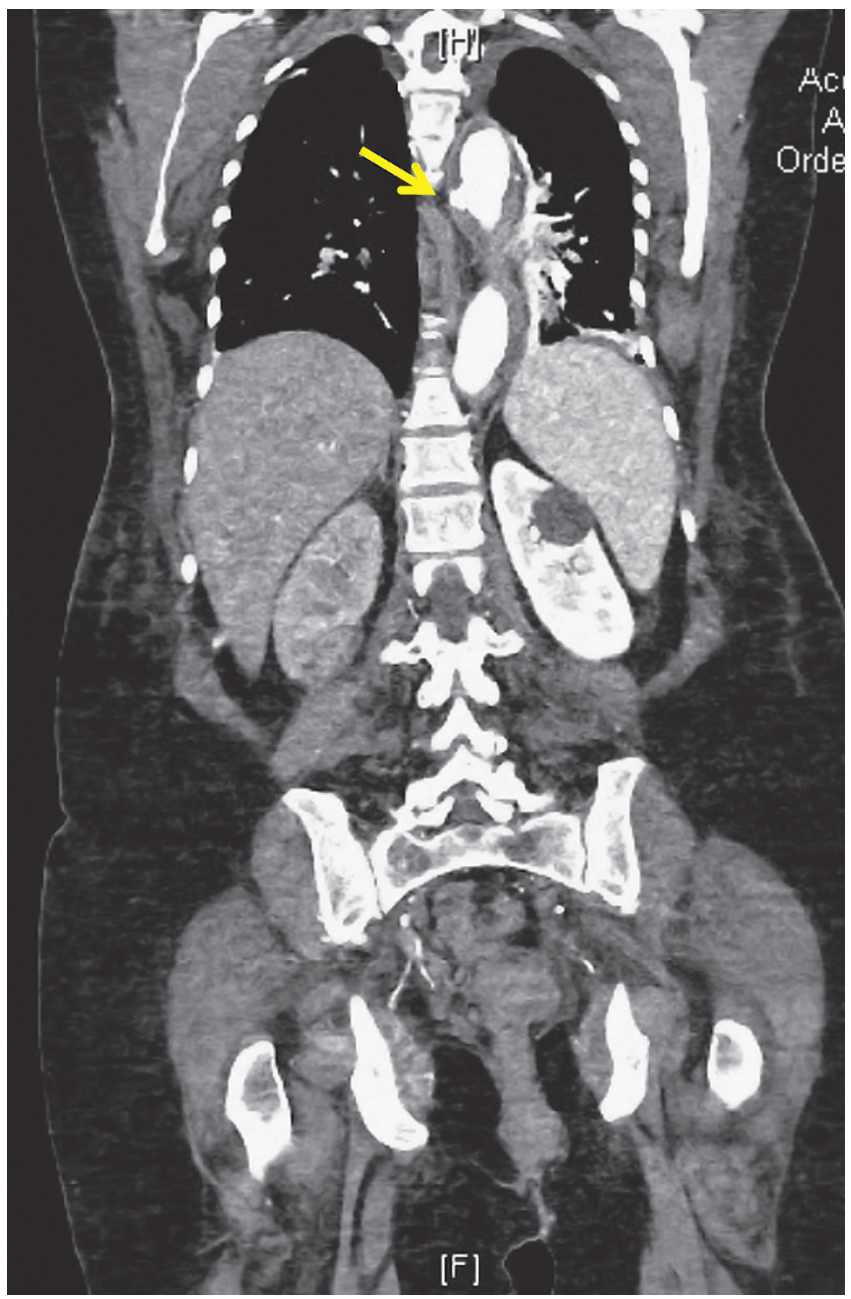

Figure 4. CT angiography coronal view demonstrating the pseudoaneurysm (yellow arrow) at the site of the intercostal branch seen in Figure 2.

median sternotomy using deep hypothermia and circulatory arrest. Due to very high mortality and morbidity associated with this approach, an alternative strategy comprised of emergency percutaneous or open distal aortic fenestration, followed by delayed complete repair, was described [3]. The latter approach re-establishes organ perfusion, allowing the patient to recover and reach the complete repair in a much better condition. By definition, this strategy, however, is not applicable in Type A IMH due to lack of proximal intimal tear. Our patient was, therefore, considered for an open conventional repair, but deemed too ill. Repeat imaging was highly suggestive that the site of origin of the acute aortic 


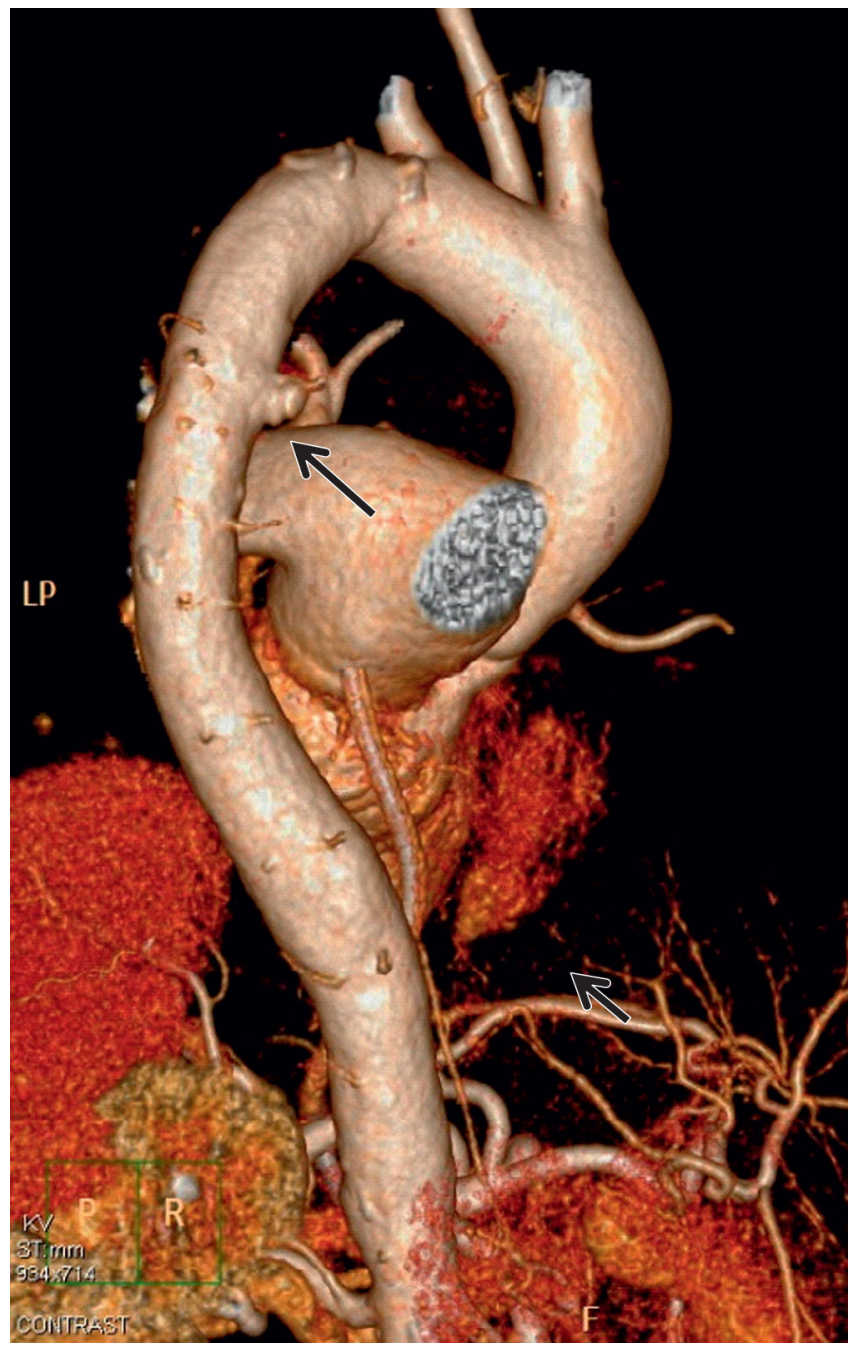

Figure 5. $C T$ angiography $3 \mathrm{D}$ reconstruction demonstrating the aortic pseudoaneurysm (black arrow) at the level of the pulmonary artery bifurcation.

pathology was, in fact, a locus minoris adjacent to a large intercostal artery in the proximal descending aorta. We therefore, elected to exclude the site using standard endovascular techniques. To minimize the risk of spinal cord injury, we covered only the suspicious segment. Two other important technical considerations included avoidance of stent-graft oversizing and balloon dilation [4]. TEVAR resulted in rapid clinical improvement and marked, sustained reverse aortic remodeling.

This case underscores the importance of frequent surveillance imaging in patients with acute aortic pathology treated medically (rather than surgically)

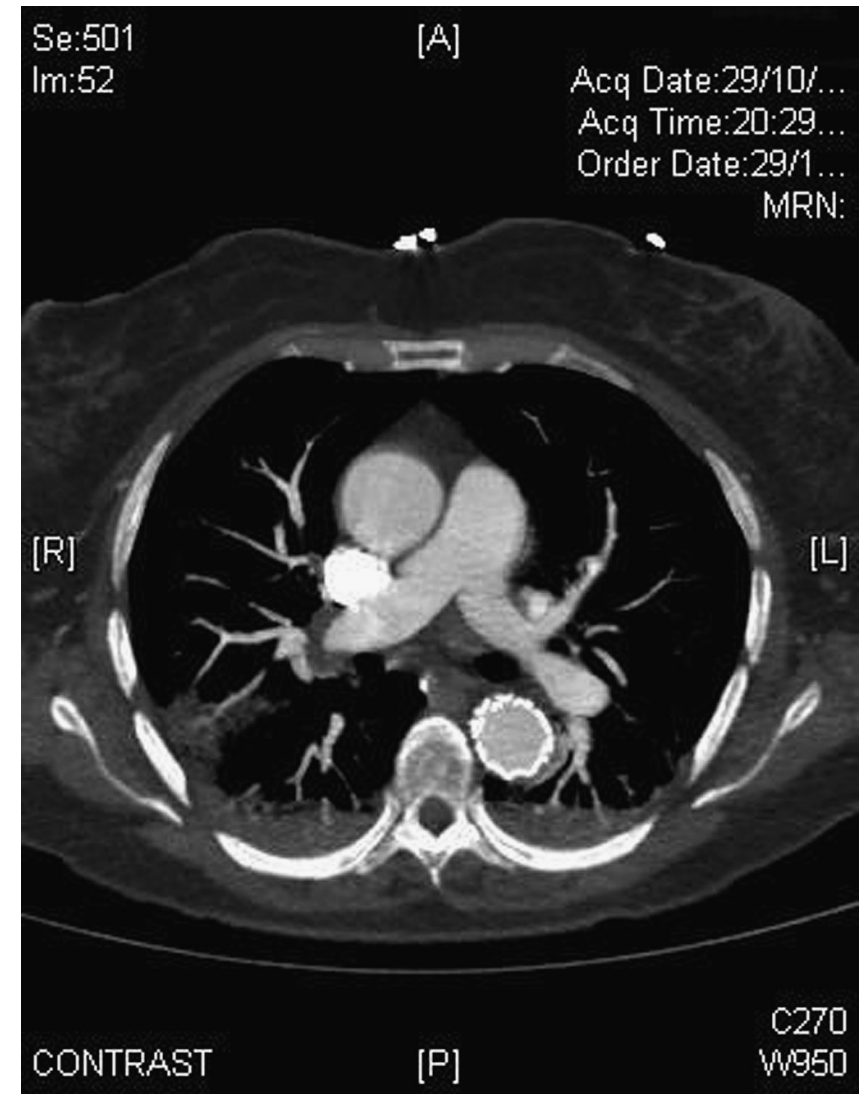

Figure 6. CT angiography axial view 9 months after the procedure: the intramural hematoma had resolved, and the stent graft is seen in the descending aorta.

aiming to better define the pathology and/or identify interval changes suggestive of pending rupture. In the current case, it enabled us to identify a very uncommon event of organ malperfusion secondary to retrograde and antegrade extension of IMH originating from a Type $B$ aortic pathology. This resulted in a dramatic shift in the operative strategy to a much less invasive approach of TEVAR.

\section{Conflict of Interest}

The authors have no conflicts of interest relevant to this publication.

Comment on this Article or Ask a Question 


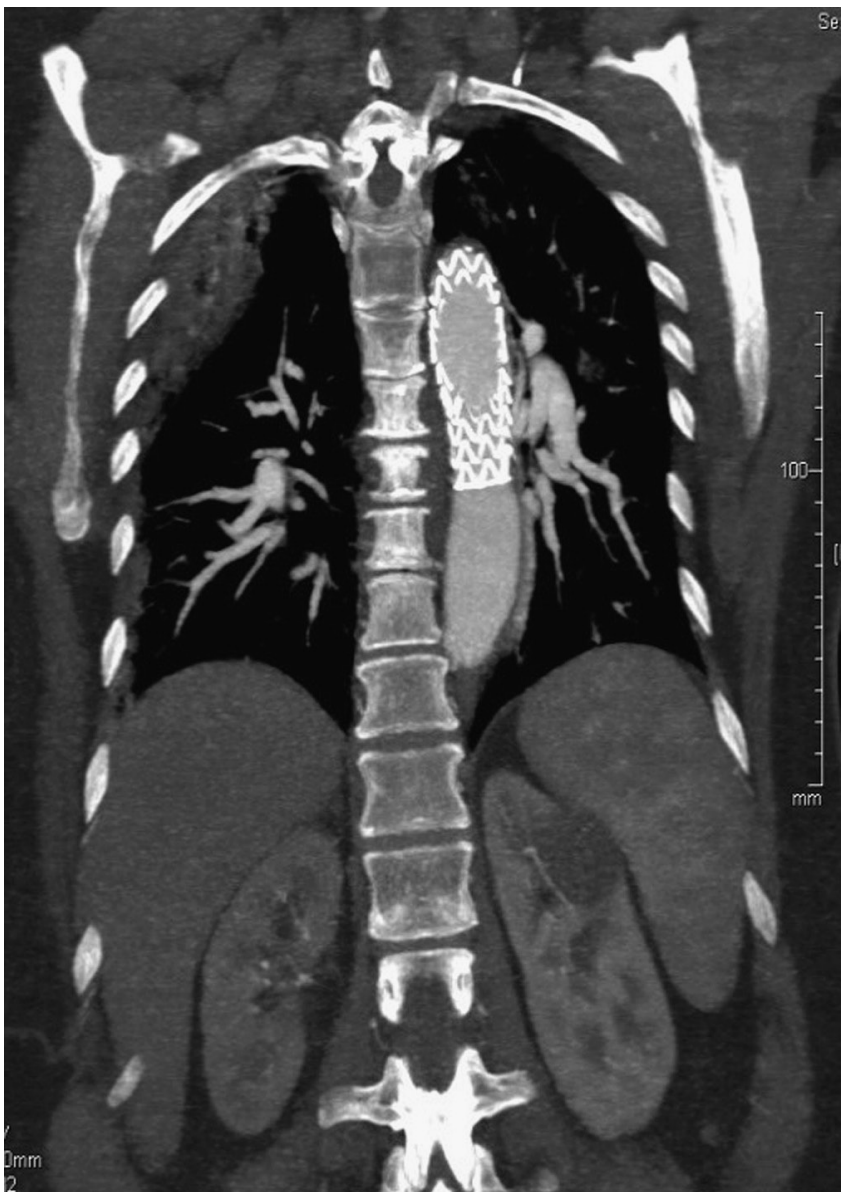

Figure 7. CT angiography coronal view 9 months after the procedure, demonstrating the stent graft in the descending aorta and the disappearance of the aortic pseudoaneurysm.

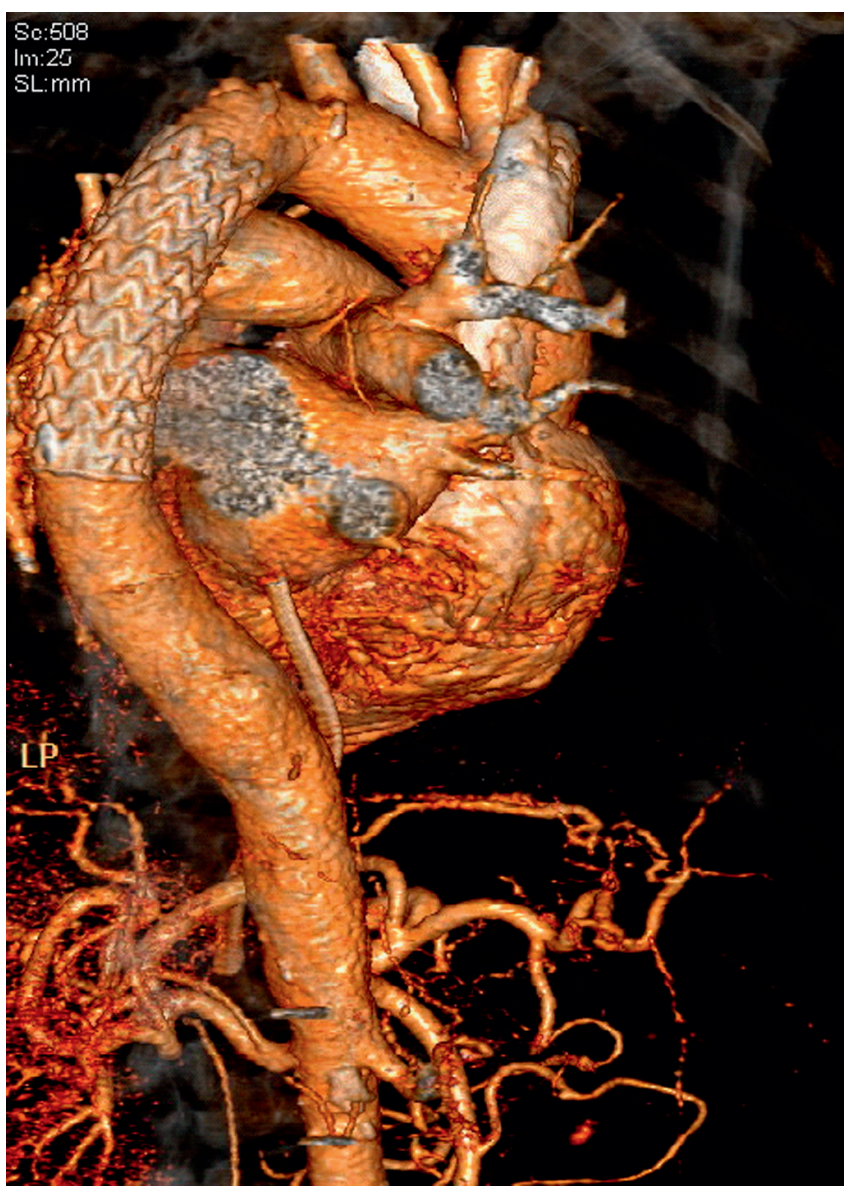

Figure 8. CT angiography $3 \mathrm{D}$ reconstruction 9 months after the procedure, demonstrating the stent graft in the descending aorta and the disappearance of the aortic pseudoaneurysm.

\section{References}

1. Krukenberg E. Beitrage zur Frage des Aneurysma dissecans. Beitr Pathol Anat Allg Pathol. 1920;67:329-351.

2. Gore I. Pathogenesis of dissecting aneurysm of the aorta. Arch Pathol Lab Med. 1952;53:142-153. PMID: 831424

3. Midulla $M$, Renaud $A$, Martinelli $T$, Koussa $M$, Mounier-Vehier C, Prat A, et al. Endovascular fenestration in aortic dissection with acute malperfusion syndrome: Immediate and late follow up. J Thorac Cardiovasc Surg. 2011; 142:66-72. DOI: 10.1016/j.jtcvs.2010.07.081

4. Zipfel B, Chiesa R, Kahlberg A, Marone EM, Rousseau H, Kaskarelis I, et al. Endovascular repair of traumatic thoracic aortic injury. Results from the relay endovascular registry for thoracic disease. Ann Thoracic Surg. 2014;97: 774-780.DOI: 10.1016/j.athoracsur.2013.09.034
Cite this article as: Levy E, Korach $A$, Rubinstein C, Gilon D, Shapira OM. Acute Type B Aortic Pathology Mimicking Acute Type A Intramural Hematoma with Organ Malperfusion. AORTA (Stamford). 2015;3(4):145-150. DOI: http://dx.doi. org/10.12945/j.aorta.2015.14.073

\section{EDITOR'S COMMENTS AND QUESTIONS}

\section{Editor's Comments}

Anneke Damberg, MD

Department of Cardiothoracic and Cardiovascular Surgery

\section{Deutsches Herzzentrum Berlin Berlin, Germany}

Levy et al. report a case of intramural hematoma/ Type $\mathrm{B}$ dissection with proximal and distal extension 
and an entry in the descending thoracic aorta, which was treated by stent graft covering of the entry.

This well presented case is of interest for the aortic surgeon since major aortic surgery was avoided by an endovascular procedure in a patient in serious condition. However, it should be pointed out that the initial decision that the patient was too sick for surgery bore a very high risk for mortality in this relatively young patient ( 56 years old) since she had a Type A extension of the dissection and an intramural hematoma, with abdominal organ malperfusion and compression of the left main coronary artery. Most surgeons would have regarded surgery as being absolutely indicated because the chances of survival with surgery still exceed by far those without [1].

Covering the entry detected on follow up CT angiogram with a stent graft led to full resolution of the hematoma. The procedure described is an elegant option to avoid major aortic surgery in this setting, even though it was risky to initially refrain from surgery or intervention in view of the malperfusion that was unlikely to resolve without therapy.

Finally, it should be mentioned that the approach presented is not new it was described in a case series by Grimm et al. [2].

\section{Editor's Questions}

\section{What happened to the LV function and AI?}

When the patient arrived to our hospital the LV function was moderately to severely reduced, it was improved to moderate after the procedure and at discharge it became mild to moderately reduced. The Al was moderate before the procedure and improved to mild Al afterwards.

\section{What happened to the left main coronary artery} compression?

When the patient first arrived to the other hospital, she had chest pain, abdominal pain, nonspecific ST-T changes, and the troponin I was not elevated, the left main artery compression was found by coronary catheterization that was done assuming that the patient had acute coronary syndrome, after the diagnosis of acute Type A IMH was established there were no signs of cardiac ischemia in the ECG, no elevation of troponin I, and the chest pain disappeared after the endovascular treatment, we assume that the distortion of the left main resolved, resulting with improvement of the LV function.

3. What happened to the liver and kidney malperfusion?

The liver functions and the liver enzymes were improved after the treatment, and were back to normal when the patient was discharged. The right kidney remained malperfused but the kidney function test were normal.

\section{References}

1. Bonser RS , Ranasinghe AM, Loubani M, Evans JD, Thalji NM, Bachet JE, et al. Evidence, lack of evidence, controversy, and debate in the provision and performance of the surgery of acute Type A aortic dissection. J Am Coll Cardiol. 2011;58(24):2455-74. DOI: 10.1016/j. jacc.2011.06.067
2. Grimm M, Loewe $C$, Gottardi R, Funovics M, Zimpfer D, Rodler $S$, et al. Novel insights into the mechanisms and treatment of intramural hematoma affecting the entire thoracic aorta. Ann Thorac Surg. 2008;86(2):453-6. DOI: 10.1016/j.athoracsur.2008.03.078 